\title{
Mortality Reduction in Acute Coronary Syndromes - How Far Are We From The Goal?
}

\section{Theodora Benedek}

University of Medicine and Pharmacy, Tîrgu Mureș, Romania

Acute coronary syndromes (ACS) are a major challenge for the healthcare in modern medicine. Many efforts are dedicated nowadays to improve the management of ACS and to facilitate an early live-saving intervention of revascularization in these cases [1].

Mortality in acute coronary syndromes remains high despite of major intervention in the healthcare systems. Implementation of regional networks dedicated to early diagnosis and treatment of acute myocardial infarction has been proved to be very effective in reduction of mortality in ST-elevation acute myocardial infarction (STEMI), and the existing networks could also be used in other acute cases, such as non ST-elevation acute myocardial infarction (NSTEMI) or unstable angina (UA). However, in these cases the networks did not show a similar efficacy as in STEMI cases, as they are mainly dedicated to early diagnosis and prompt intervention of STEMI cases, fact that leaves NSTEMI and UA cases somehow neglected.

Different factors have been proved to be associated with in-hospital or long-term mortality in ACS. Gender and age are major factors that can influence mortality, recent studies demonstrating that mortality is significantly higher in women under the age of 60 , while in women older than 60 the existing comorbidities have a significant impact on the outcome of revascularization in the long term [2].

Comorbidities are also strong predictors of death after PCI. Renal failure proved to be strongly associated with a higher risk of death following PCI in acute cases, in a significantly higher extent than in stable coronary artery disease. A recent study demonstrated a double relative risk associated with renal failure in STEMI cases (odds ratio 5.3) as compared with ACS cases (odds ratio 2.1), and with stable coronary artery disease (odds ratio 2.3) [3].

Heart failure and cardiogenic shock at presentation have also been proved to be associated with a significantly higher death rate, in both STEMI and NSTEMI cases. At the same time, heart failure is a strong predictor of acute kidney failure in patients with STEMI undergoing primary PCI [4]. Other studies demonstrated the impact of multivessel disease on the outcome of the revascularization procedure, being generally accepted that the presence of a multivessel disease has a negative impact on the survival of ACS patients [5].

A study published in this issue demonstrates that in ACS cases, in-hospital mortality is correlated with a multitude of factors, including age, gender, troponin levels, necessity of intra-aortic balloon pump insertion or cardiogenic shock at presentation, multivascular disease, out of hospital resuscitation and the presence of impaired renal function, or comorbidities such as arterial hypertension and diabetes [6]. Interestingly, this study shows that older patients require a longer duration of stay in the intensive care unit as compared with younger patients (73.0 \pm 8.5 vs $64.4 \pm 11.2, p=0.007$ ), demonstrating that age is correlated not only with mortality, but also with the risk of complications in ACS.

Another interesting observation of the study was that in STEMI cases, culprit lesions located in the left anterior descending artery were present in $44.4 \%$ of patients presenting in cardiogenic shock, while they were present in only $21.7 \%$ of patients in good hemodynamic condition at presentation, without cardiogenic shock. However, in NSTEMI patients, the localization of the culprit lesion in 
the left main artery significantly correlated with the development of cardiogenic shock, which indicates that culprit lesions located in the left main and causing a NSTEMI are more likely to result in development of a cardiogenic shock, which is probably also caused by the complexity of coexisting morbidities and the multivascular disease frequently encountered in these complex cases.

However, the main issue raised by this study is how to obtain a more significant reduction in mortality in these cases. Primary percutaneous intervention in STEMI is associated with a mortality as low as $3-5 \%$ in experienced

\section{REFERENCES}

1. Ghauharali-Imami S, Bax M, Haasdijk A, et al. The impact of gender on long-term mortality in patients with multivessel disease after primary percutaneous coronary intervention. Neth Heart J. 2015;23:592-599.

2. Loncar G, Barthelemy O, Berman E, et al. Impact of renal failure on all-cause mortality and other outcomes in patients treated by percutaneous coronary intervention. Arch Cardiovasc Dis. 2015;108:554-562.

3. Matejka J, Varvarovski I, Rozsival V, et al. Heart failure is the strongest predictor of acute kidney injury in patients undergoing primary percutaneous coronary intervention for ST-elevation myocardial infarction. Kardiol Pol. 2015; doi: 10.5603/KP.a2015.0115. [Epub ahead of print]

4. Jang JS, Spertus JA, Arnold SV, et al. Impact of Multivessel centers and this should represent the goal for all ACS cases, including NSTEMI and UA. All the efforts should be made for reaching this goal and eventually for improving furthermore the results of revascularization [7]. A superior network organization, in order to ensure a superior usage of the existing STEMI networks in NSTEMI or UA cases, can represent a major target for intervention. This would lead to a more rapid intervention, before severe complications (such as cardiogenic shock) occur, thus preventing the complications associated with cardiogenic shock and leading to a significant reduction in mortality.

Revascularization on Health Status Outcomes in Patients With ST-Segment Elevation Myocardial Infarction and Multivessel Coronary Artery Disease. J Am Coll Cardiol. 2015;66:2104-2113.

5. Barcan A, Kovacs I, Blendea C, Orzan M, Chitu M. Factors associated with in-hospital mortality in patients with acute coronary syndrome. Journal Cardiovasc Emerg. 2015:1(2):6874 .

6. Ramanath VS, Eagle KA. Evidence-based medical therapy of patients with acute coronary syndromes. Am J Cardiovasc Drugs. 2007;7:95-116.

7. Schehter M, Goldenberg I, Matetztki Slomi. Trends in mortality rates from acute coronary syndrome octogenarian patients in Israel during the period 2003-2013. J Am Coll Cardiol. 2015;65(10_S). 